\title{
ORAL HEALTH: A PRIMER
}

\author{
Matthew N. O. Sadiku', Tolulope J. Ashaolu²*, and Sarhan M. Musa1 \\ ${ }^{1}$ Roy G. Perry College of Engineering, Prairie View A\&M University, Prairie View, TX, USA \\ ${ }^{2}$ College of Food Science, Southwest University, Tiansheng Road Beibei District, Chongqing, 400715, P.R. China \\ E-mail: sadiku@ieee.org; ashaolut@gmail.com; smmusa@pvamu.edu
}

*Corresponding author details: Dr. Tolulope J. Ashaolu, ashaolut@gmail.com

\begin{abstract}
Health is a quality of life with social, mental, emotional, spiritual, and physical functions. Good oral health is key to good overall health. It can have a significant impact on the overall health and well-being of our citizens. It affects people physically and mentally and influences how we grow, enjoy life, look, speak, chew, taste food, and socialize. Oral diseases affect our well-being throughout life. Taking good care of our mouth, teeth and gums is a worthy goal. This paper discusses oral health and how bad oral health can be prevented.
\end{abstract}

Keywords: oral health; dental health; oral health inequalities

\section{INTRODUCTION}

The human body is a complex system that interconnects all the parts. One part of the body can have an impact on other part. Our mouth may be regarded as a window into the health of our body. We use our teeth and mouths a lot and many things can go wrong. A healthy mouth can be a great asset. As shown in Figure 1, the teeth contain a vital pulp and are supported by the periodontal ligament [1]. Our teeth play many functions in our lives. They help us chew and digest food, communicate with others, smile, and give our face its shape. In light of this, it is expedient to give our oral health the best care possible.

Good health is a major resource for social, economic, and personal development. Oral health is an integral part of general health and wellbeing. Poor oral health is a significant public concern in the United States. It has vast implications on quality of life, affecting an individual's psychological health, ability to socialize, feelings of social wellbeing, and enjoyment of life. Oral health is regarded as the silent epidemic by Regina M. Benjamin, the Surgeon General [2]. Good oral health enables individuals to communicate effectively and enjoy foods. Health promotion puts health on the agenda of policy makers in all sectors. Oral health is an integral part of our overall health and well-being. The word "oral" refers to the mouth, which includes the teeth, the gums, and their supporting tissues. Oral health refers to the health of the teeth, gums, and the entire oral-facial system. It is related to well-being and quality of life.

\section{IMPORTANCE OF ORAL HEALTH}

Oral health is defined as the ability to speak, smile, smell, taste, touch, chew, swallow and convey a range of emotions through facial expressions with confidence and without pain, discomfort and disease of the craniofacial complex [3]. Studies show that poor oral health has a detrimental impact on general health. It can be detrimental and costly. Many systemic diseases and conditions have oral manifestations.
Oral health is important to our general health and wellbeing and can be achieved by all Americans. Good oral health contributes to a person's ability to speak, smile, smell, taste, touch, chew, swallow, and make facial expressions. Poor oral hygiene can lead to gum disease. It has been associated with heart disease, cancer, and diabetes. It can impair our ability to eat and speak properly, causing pain and bad breath.

Oral health involves the prevention of dental diseases. The major factors that affect oral health include individual biology and genetics, the environment, personal behaviors and lifestyle, access to care, and the organization of healthcare. Access to oral healthcare is dictated by factors such as education level, income, race, age, sex, and ethnicity.

\section{TYPES OF ORAL DISEASES}

Oral diseases pose a serious health concern. They affect people throughout their lifetime, causing pain, discomfort, and even death. You may experience at least one dental problem during your lifetime. Common oral diseases include [4]:

- Cavities: These are areas of the tooth that have been permanently damaged, sometimes with holes. Cavities occur when bacteria, food, and acid coat your teeth and form a plaque.

- Gum disease: This is an infection or a destructive inflammation of the gums and it is also called gingivitis. It is usually the result of plaque building up on your teeth due to poor brushing and flossing habits. Untreated gingivitis can lead to periodontitis, a more serious infection. Figure 2 show an example of gum disease (gingivitis) [5].

- Periodontitis: This is the more advanced stage of gum or gingivitis. Factors that contribute to gingivitis and periodontitis include smoking, poor brushing habits, snacking on sugary foods and drinks.

- Cracked teeth: A tooth can crack or break due to an injury to the mouth or chewing hard foods.

- Sensitive teeth: Your teeth are sensitive when you feel pain or discomfort after taking cold or hot foods or drinks 
- Oral cancer: This includes cancer of the gums, tongue, lips, or cheek. Smoking or chewing tobacco is the biggest risk factor for oral cancer.

- Dry mouth: Some medications can affect the amount of saliva in your mouth.

- $\quad$ Mouth Sores: There are many types of mouth sores. It is usually nothing to worry about and will disappear on its own. But if it lasts more than two weeks, you should see your dentist.

- Noma: This is a severe gangrenous disease of the mouth and the face. It mostly affects children between the ages of 2 and 6 years suffering from malnutrition, mostly in many developing countries in Africa and Asia.

- Dental erosion: Dental erosion is the progressive, irreversible loss of dental hard tissue.

- Dental caries: This is commonly known as tooth decay. It affects people of all ages, causing tooth loss if not treated. It is the most common chronic disease of in school-aged children. Untreated dental caries remains the most prevalent oral condition worldwide.

Other serious health problems caused by bad oral health include cardiovascular disease, dementia, respiratory infections, diabetes, pregnancy complications, infertility, erectile dysfunction, kidney disease, and rheumatoid arthritis. Some of these diseases are a life-and-death matter. Oral diseases are the fourth most expensive diseases to treat in some countries.

\section{PREVENTION OF ORAL DISEASE}

Maintaining healthy teeth and gums is a lifelong commitment. Everyone has a role to play in preventing, improving, and promoting oral health. Achieving oral health requires the combination of efforts from individuals, healthcare professionals (particularly dental practitioners), and community-based activities.

Prevention of disease is regarded as the key to maintaining good overall health. It should be a primary goal of any society that hopes to provide a decent quality of life for its citizens. Oral health prevention strategies aim to prevent oral health disease before it occurs or to reduce the impact of oral health disease. Many oral diseases can be prevented through early detection and intervention. The prevention of oral disease and disability has been investigated by scientists, dentists, dental hygienists, and other health professionals. The existing prevention measures can be adopted by everyone to improve oral health and prevent disease. These measures include daily oral hygiene procedures, tobacco control and cessation programs, and communitybased preventive programs [6].

Consider taking care of your oral health as an investment in your overall health. You can keep your teeth healthy and reduce oral disease by [4]:

- $\quad$ brushing your teeth with fluoride toothpaste at least twice a day

- flossing your teeth at least once a day

- using mouthwash to remove food particles left after brushing and flossing

- replacing your toothbrush every three or four months or sooner

- promoting a well-balanced diet high in fruit and vegetables

- favoring fluoridated water as the main drink

- limiting your intake of sugar

- reducing alcohol consumption

- $\quad$ eating a diet high in fruits and vegetables
- avoiding smoking cigarettes or chewing tobacco products

- $\quad$ seeking professional dental care

- $\quad$ scheduling regular dental checkups and cleanings

\section{BENEFTIS}

Good oral health is a combination of proper daily maintenance (brushing and flossing) along with regular visits to your dental care professional. Seeing a dentist regularly helps to keep your mouth in top shape and allows your dentist to watch for developments that may point to other health issues.

Good oral health can have so many wonderful lifechanging benefits. Practicing good oral health is essential for maintaining a healthy mouth, teeth, and gums. It will also help your appearance and quality of life. Good oral health can really prevent certain diseases from occurring. It can help you steer clear of bad breath (halitosis).

\section{CHALLENGES}

Although significant improvements have made in our nation's oral health, some challenges remain. The growing globalization, urbanization, industrialization, and inequalities hinder the promise of desirable changes in oral health. There are multiple barriers to widespread adoption of oral health care, including cost, absence of dental insurance, deficiency of dental services, and lack of oral health literacy [7]. Unfortunately, some Americans do not have access to preventive programs and are vulnerable to having oral diseases [8]. The expenses associated with oral health problems are costly to the individual and society. Individuals should be responsible for their conduct, while society is largely responsible for their conditions.

The dental workforce is declining and may not meet the emerging demands of the society. There is a lack of racial ethnic diversity in dental workforce. A decline in the number of faculty and researchers may seriously affect the quality of dental education. The integration of oral and general health programs is still lacking. Little or no time is devoted to teaching oral health to nondental health professionals.

Figure 3 shows the main determinants of health that shows that overarching societal factors operate through people's living and working conditions to influence health [9]. Lack of access to dental care for all ages and ethnic groups remains a public health challenge. Oral diseases disproportionally affect the poor and sociallydisadvantaged members of society. The public health infrastructure for oral health is insufficient to address the needs of disadvantaged groups. Global oral health inequalities constitute a challenge that must be addressed in the context of global public health.

\section{CONCLUSION}

Proper oral health is essentially maintaining healthy teeth. Oral health is vital to total health and satisfactory quality of life. The negative impact of inadequate care for teeth, gums, bite, and jaws can move beyond the mouth to affect overall physical health. Regular brushing, flossing, and dentist visits can help prevent disease in our mouth.

Prevention of diseases is the most cost effective approach and has the greatest impact on the society. Promotion of good oral health is a fundamental need across the globe. Educating ourselves on the common oral health problems and their causes can also go a long way in prevention. Oral health should also be promoted and discussed in schools to help children and adolescents develop correct oral health behaviors. 
Web sites that provide information about good oral health practices, prevention, treatment options, and curriculum resources for teachers are compiled in [10]. More information about oral health can be found in the books [11-17] and in the following journals devoted to it:

- $\quad$ The Journal of the American Dental Association

- British Medical Journal

\section{REFERENCES}

[1] R. Holt, G. Roberts, and C. Scully, "ABC of oral health: Oral health and disease," British Medical Journal, vol. 320, no. 7250, June 2000, pp. 1652-1655.

[2] R. M. Benjamin, "Oral health: The silent epidemic," Public Health Reports, vol. 125, no. 2, March/April 2010, pp. 158-159.

[3] N. J. Wilson et al., "Oral health status and reported oral health problems in people with intellectual disability: A literature review," Journal of Intellectual \& Developmental Disability, vol. 44, no. 3, 2019, 292304.

[4] "Everything you need to know about dental and oral health," Unknown Source.

[5] H. Silk et al., "Oral health during pregnancy," https://www.aafp.org/afp/2008/0415/p1139.html

[6] "2000 Surgeon General's report on oral health in America,"

https://www.nidcr.nih.gov/news-events/2020surgeon-generals-report-oral-health

[7] L. Aungst and B. A. Swan, 'Examining oral health education for nondental providers," The Journal for Nurse Practitioners, vol. 16, no. 6, June 2020, pp. 470-473.

[8] "Oral health," https://www.healthypeople.gov/2020/topicsobjectives/topic/oral-health

[9] A. Sheiham et al., "Global oral health inequalities: Task group - Implementation and delivery of oral health strategies," Advances in Dental Research, vol. 23, no. 2, May 2011, pp. 259-267. 2011
[10] J. E. Cox, "Oral health information on the Internet," Journal of Consumer Health on the Internet, vol.12, no. 2, 2008, pp. 131-142.

[11] Oral health surveys: basic methods. Geneva: Switzerland: World Health Organization, $5^{\text {th }}$ edition. 2013.

[12] Institute of Medicine, Advancing Oral Health in America. National Academies Press, 2012.

[13] M. S. Virdi (ed.), Emerging Trends in Oral Health Sciences and Dentistry. Rijeka, Croatia: InTech, 2015.

[14] A. Chattopadhyay, Oral Health Epidemiology: Principles and Practice. Sudburry, MA: Jones and Bartlett Publishers, 2011.

[15] I. C. Bagewitz, Prosthodontics and Oral Health-Related Quality Of Life. VDM Verlag, 2008.

[16] J. Bertness and K. Holt (eds.), Promoting Oral Health in Schools: A Resource Guide. Washington, DC: National Maternal and Child Oral Health Resource Center, 2009.

[17] D. M. O'Mullane and C. Dawes, Saliva and Oral Health. Stephen Hancocks Limited, 2004.

\section{AUTHORS}

Matthew N.o. Sadiku is a professor in the Department of Electrical and Computer Engineering at Prairie View A\&M University, Prairie View, Texas. He is the author of several books and papers. His areas of research interests include computational electromagnetics and computer networks. He is a fellow of IEEE.

Tolulope J. Ashaolu works at Southwest University. He is the author of several papers and a book. His research interests include functional foods and food microbiology.

Sarhan M. Musa is a professor in the Department of Electrical and Computer Engineering at Prairie View A\&M University, Prairie View, Texas. He has been the director of Prairie View Networking Academy, Texas, since 2004. He is an LTD Sprint and Boeing Welliver Fellow. His areas of research interests include computational electromagnetics and computer networks.

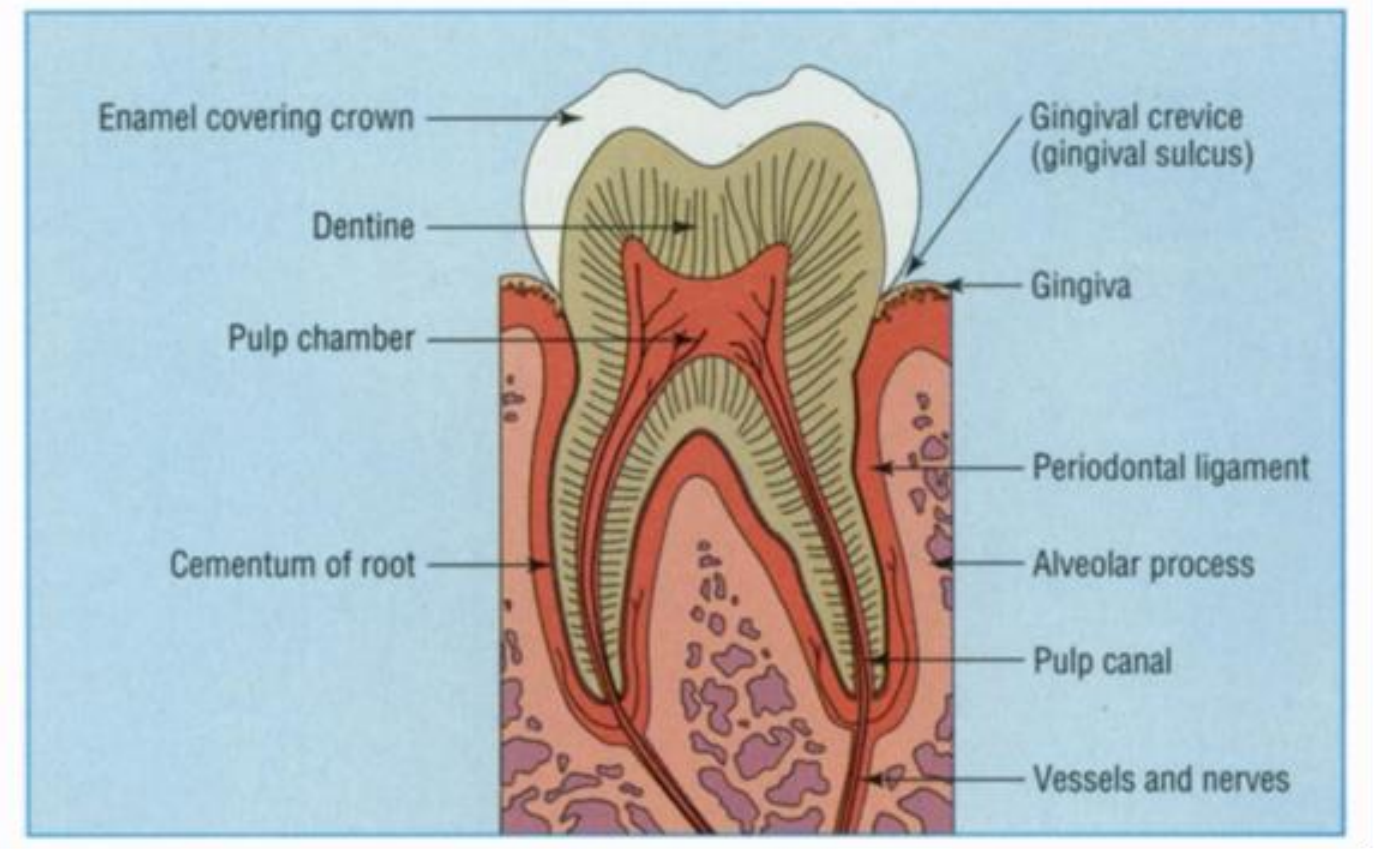

FIGURE 1: A TOOTH AND SUPPORTING STRUCTURES [1] 


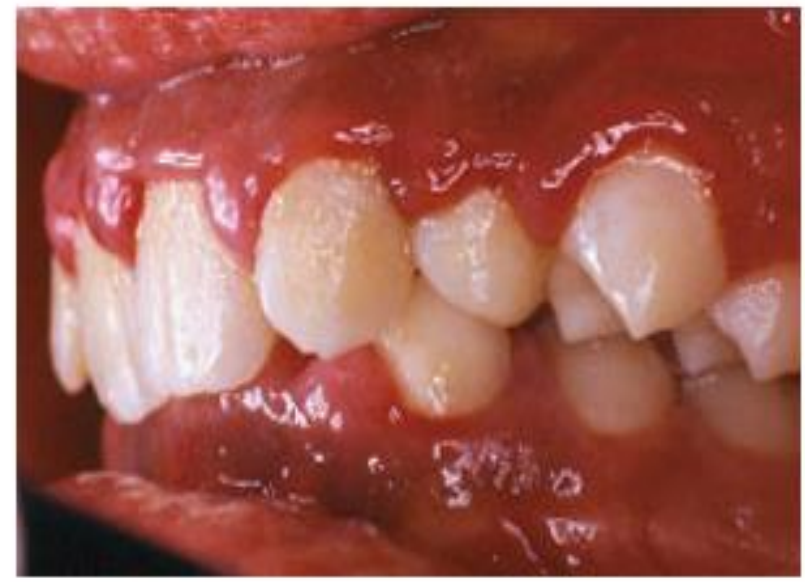

FIGURE 2: AN EXAMPLE OF GUM DISEASE (GINGIVITIS) [5].

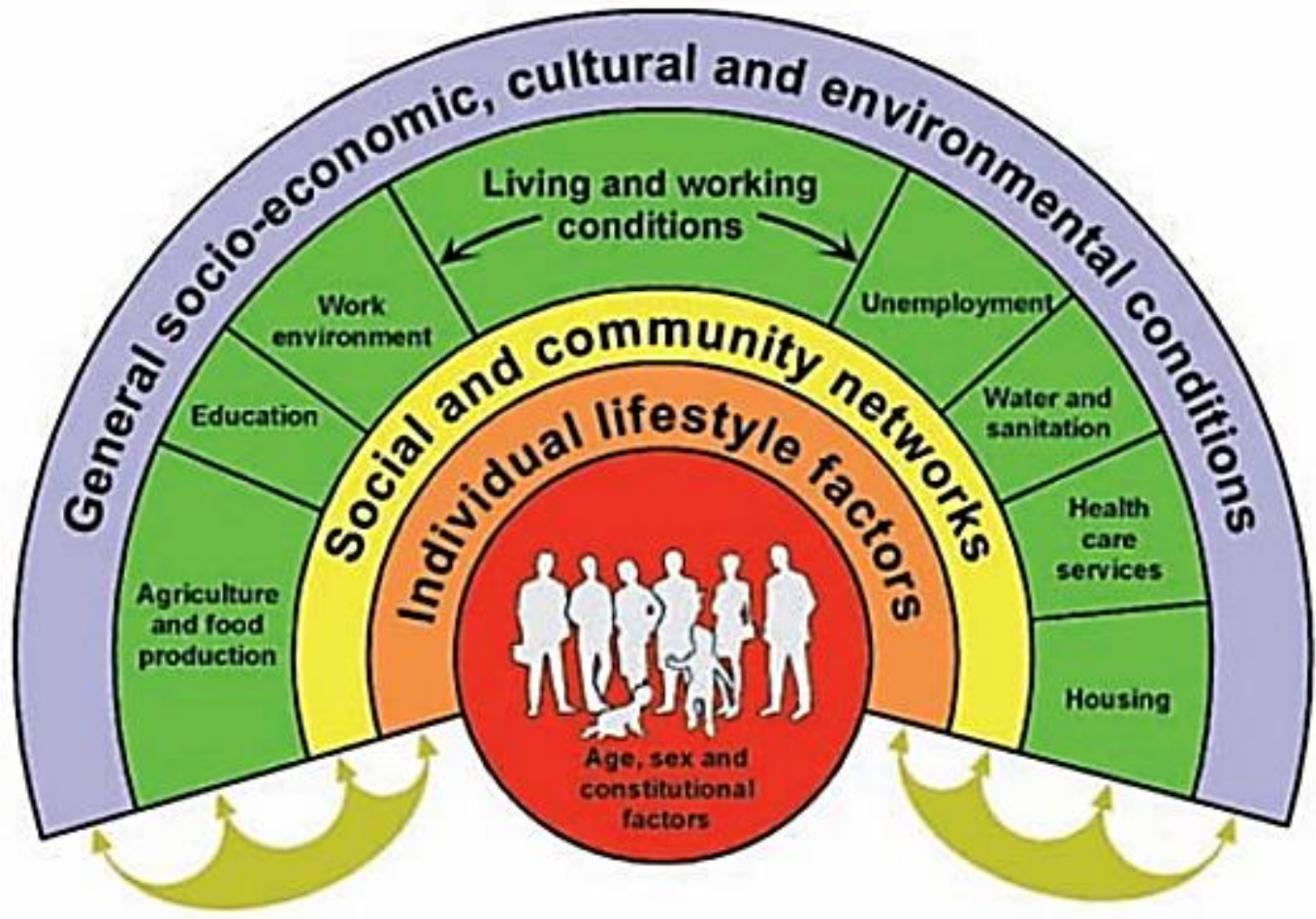

FIGURE 3: THE MAIN DETERMINANTS OF HEALTH THAT SHOWS THAT OVERARCING SOCIETAL FACTORS OPERATE THROUGH PEOPLE'S LIVING AND WORKING CONDITIONS [9]. 